\title{
Collective States of Non-Abelian Quasiparticles in a Magnetic Field
}

\section{Citation}

Levin, Michael and Bertrand I. Halperin. 2009. Collective states of non-Abelian quasiparticles in a magnetic field. Physical Review B 79(20): 205301.

\section{Published Version}

http://dx.doi.org/10.1103/PhysRevB.79.205301

\section{Permanent link}

http://nrs.harvard.edu/urn-3:HUL.InstRepos:3110941

\section{Terms of Use}

This article was downloaded from Harvard University's DASH repository, and is made available under the terms and conditions applicable to Open Access Policy Articles, as set forth at http:// nrs.harvard.edu/urn-3:HUL.InstRepos:dash.current.terms-of-use\#OAP

\section{Share Your Story}

The Harvard community has made this article openly available.

Please share how this access benefits you. Submit a story.

Accessibility 


\title{
Collective states of non-Abelian quasiparticles in a magnetic field
}

\author{
Michael Levin ${ }^{1,2}$ and Bertrand I. Halperin ${ }^{2}$ \\ ${ }^{1}$ Department of Physics, University of California, Santa Barbara, California 93106, USA \\ ${ }^{2}$ Department of Physics, Harvard University, Cambridge, Massachusetts 02138, USA \\ (Received 4 December 2008; revised manuscript received 1 April 2009; published 4 May 2009)
}

\begin{abstract}
Motivated by the physics of the Moore-Read $\nu=1 / 2$ state away from half filling, we investigate collective states of non-Abelian e/4 quasiparticles in a magnetic field. We consider two types of collective states: incompressible liquids and Wigner crystals. In the incompressible liquid case, we construct a natural series of states which can be thought of as a non-Abelian generalization of the Laughlin states. These states are associated with a series of hierarchical states derived from the Moore-Read state- the simplest of which occur at filling fraction $8 / 17$ and 7/13. Interestingly, we find that the hierarchical states are Abelian even though their parent state is non-Abelian. In the Wigner crystal case, we construct two candidate states. We find that they, too, are Abelian - in agreement with previous analysis.
\end{abstract}

DOI: 10.1103/PhysRevB.79.205301

PACS number(s): 73.43.Cd, 71.10.Pm

\section{INTRODUCTION}

The Moore-Read Pfaffian state ${ }^{1}$ (or its particle-hole conjugate, the anti-Pfaffian ${ }^{2,3}$ ) is believed to be a good candidate for the observed $\nu=5 / 2$ quantum Hall plateau. ${ }^{4}$ This possibility is particularly exciting since the quasiparticle excitations in this state carry non-Abelian statistics. Much work has been devoted in understanding the basic physical properties of the Pfaffian state. However, one aspect of the Pfaffian state has received less attention-the physics of this state away from half filling.

To understand the basic issue, consider an idealized, perfectly clean fractional quantum hall $(\mathrm{FQH})$ system whose ground state at half filling is the Pfaffian state. Suppose that the magnetic field is adjusted so that the filling fraction is slightly less than $1 / 2$. In this case, we expect that the ground state will be given by the Pfaffian together with a small but finite density of $e / 4$ quasiholes.

This unusual physical system motivates a general question: what kinds of collective states can arise from a finite density of non-Abelian $e / 4$ quasiholes in a magnetic field? This is the main subject of this paper. We consider two basic kinds of collective states. The first kind of state is a Wigner crystal of quasiholes. Such a state is natural if the dominant interaction between the quasiholes is the repulsive Coulomb force, and the quasiholes are sufficiently dilute. Thus it is a good candidate for a clean FQH system at filling $\nu$ very close to $5 / 2$. The second possibility we consider is that the quasiholes form an incompressible liquid. This is perhaps less relevant to a physically realistic $\nu=5 / 2$ system, but it is interesting conceptually. In the context of the Pfaffian state, such an incompressible liquid would correspond to a hierarchical quantum Hall state-but unlike the usual FQH hierarchy construction, ${ }^{5,6}$ it would be built out of non-Abelian anyons.

One way that quasiholes can form an incompressible liquid is if they pair to form charge $e / 2$ Abelian anyon boundstates, as was proposed by Bonderson and Slingerland. ${ }^{7}$ These Abelian anyons can then form Laughlin-type incompressible states. The result is a series of hierarchical states which carry non-Abelian statistics similar to the Pfaffian state.
In this paper, we construct a different kind of condensate - one made up of individual non-Abelian quasiholes rather than Abelian anyon molecules. The motivation for this is conceptual: it is not obvious what kinds of incompressible liquids can form from non-Abelian anyons. Currently, we do not have good reason to decide whether this type of condensate, the paired condensate in Ref. 7, or something entirely different is most likely in a realistic $\nu=5 / 2$ system. Our philosophy is rather that each of these possibilities can occur in principle (depending on the details of the electron interaction) and therefore merits consideration.

Our starting point is a model where the quasihole interaction is short range, two-body, and repulsive, and the quasiholes are in their lowest effective Landau level. Our main result is that we construct a series of incompressible liquids that are good candidate ground states for such a model. These states can be regarded as a non-Abelian generalization of the Laughlin states. ${ }^{8}$ In the context of the Pfaffian state near half filling, these states are associated with a series of hierarchical FQH states at filling fractions $8 m /(16 m+1)$ $=8 / 17,16 / 33, \ldots$. Interestingly, we find that these states are actually Abelian quantum Hall states-even though they are derived from the non-Abelian Pfaffian state. We find that these states are distinct from the usual Jain states ${ }^{9}$ (or equivalently hierarchical states $\left.{ }^{10}\right)$ at filling fractions $8 m /(16 m+1)$ but are equivalent to hierarchical states derived from the strong pairing $\nu=1 / 2$ state. $^{11} \mathrm{We}$ also consider incompressible liquids of $e / 4$ quasiparticles. We again find a series of states-in this case at filling fractions $(8 m-1) /(16 m-3)$ $=7 / 13,15 / 29 \ldots$. These states are also Abelian and distinct from the Jain states. They are equivalent to hierarchical states derived from the $331 \nu=1 / 2$ state. ${ }^{11,12}$

In addition, we consider the problem of a Wigner crystal of quasiholes. The physics of this system is nontrivial due to the non-Abelian statistics of the quasiholes. Even after the quasiholes localize at certain positions in space, there are still $2^{N_{\mathrm{qh}} / 2-1}$ nearly degenerate quasihole states coming from their non-Abelian statistics. To fully specify the ground state, one needs to specify a state in this $2^{N_{\mathrm{qh}} / 2-1}$ dimensional Hilbert space. Previous analysis of an exactly soluble (but simplified) model yielded a particular ground state which, in the context of the Pfaffian state, turned out to be an Abelian 
state $^{13}$ (see also Ref. 14 and in the disordered case, Ref. 15). More specifically, this state turned out to be in the same universality class as the strong pairing state. In this paper, we simply look for natural candidate states in the $2^{N_{\mathrm{qh}} / 2-1}$ dimensional Hilbert space. We find two of them, both Abelian. One is in the strong pairing universality class, and one is in the 331 universality class. While our approach is not well controlled like the analysis in Ref. 13, it has the advantage that it can be generalized to a Wigner crystal of Read-Rezayi quasiholes ${ }^{16}$ —a system which appears less amenable to the exact analysis of Ref. 13.

Currently, there is no experimental evidence for the above series of hierarchical states. No plateaus have been found at any of the filling fractions $8 / 17,7 / 13$, etc. Therefore, we feel the main contribution of this paper is conceptual. We hope that our discussion reveals some of the basic phenomena in the many-body physics of non-Abelian anyons.

The paper is organized as follows. In Secs. II and III we consider the idealized problem of a gas of pointlike nonAbelian anyons in a magnetic field. We give a quantummechanical description of this system in Sec. II, and we propose a series of candidate incompressible states in Sec. III. In Secs. IV and V we apply these results to the Pfaffian state. We construct a series of hierarchical quantum Hall states built out of the non-Abelian quasiholes and examine their properties. In Sec. VI we repeat the analysis for the nonAbelian quasiparticle and construct another series of hierarchical states. In Secs. VII and VIII we consider higher level hierarchical states and states derived from the anti-Pfaffian state. Finally, in Sec. IX we apply our approach to the problem of a quasihole Wigner crystal.

\section{QUANTUM MECHANICS OF NON-ABELIAN ANYONS}

In this section, we address a preliminary question-how to formulate quantum mechanics for pointlike non-Abelian anyons. We focus on the case of non-Abelian anyons with the same statistics as the quasihole in the Pfaffian state, as that is the case that is relevant to our later analysis. We also assume the particles are hardcore so as to avoid singularities when particles occupy the same point in space.

To begin, consider a system of four such non-Abelian anyons and assume that the anyons are at fixed positions $r_{1}, r_{2}, r_{3}, r_{4}$. Because of the non-Abelian statistics of the anyons, the four anyon system can be in two states. Thus, the Hilbert space for this system is two dimensional, and the wave functions that describe the states are two component row vectors $\left(\Psi_{1}, \Psi_{2}\right)$. The Hamiltonian for such a system can be any $2 \times 2$ Hermitian matrix.

Now consider the case where the anyons are free to move. A natural guess is that the appropriate wave functions for this system are two component row vectors of functions, $\left(\Psi_{1}\left(r_{1}, r_{2}, r_{3}, r_{4}\right), \Psi_{2}\left(r_{1}, r_{2}, r_{3}, r_{4}\right)\right)$ while the Hamiltonian is the usual kind of differential operator. This is essentially correct, but we need to take account of the Berry phases associated with the exchange of the anyons in some way. There are two ways to do this. One can either add a nonlocal Chern-Simons interaction to the Hamiltonian, or one can modify the Hilbert space so that the allowed wave functions are multivalued.
In this paper, we will use the second approach. We take our wave functions $\left(\Psi_{1}, \Psi_{2}\right)$ to be multivalued on the four particle configuration space $\left\{\left\{r_{1}, r_{2}, r_{3}, r_{4}\right\}: r_{i} \neq r_{j}\right\}$ - or more accurately, single valued on a Riemann sheet covering this space. We impose a constraint on the wave functions analogous to the antisymmetry constraint on fermionic wave functions. The constraint is defined as follows. Recall that for every possible braid or exchange of the four anyons, there is a corresponding $2 \times 2$ unitary matrix $U_{\alpha \beta}$ which describes the effect of this exchange on the two degenerate states: $|\alpha, \mathrm{ex}\rangle=\Sigma_{\beta} U_{\alpha \beta}|\beta\rangle$. We constrain our wave functions to have the property that under such an exchange, the $\left(\Psi_{1}, \Psi_{2}\right)$ transform according to this unitary matrix: $\Psi_{\alpha, \text { ex }}=\Sigma_{\beta} \Psi_{\beta} U_{\beta \alpha}^{*}$. Also, since the anyons are hardcore particles, we impose the boundary condition that $\Psi_{1,2} \rightarrow 0$ as $r_{i} \rightarrow r_{j}$.

In the multivalued wave-function approach, the Berry phases are taken care of by the choice of Hilbert space. The Hamiltonian for a four-anyon system is therefore no different from a four-boson or four-fermion system (at the level of a differential operator). For example, the appropriate Hamiltonian for describing four anyons with quadratic dispersion, charge $q$, mass $m$, and subject to a uniform magnetic field $B$ in the $\hat{z}$ direction is simply

$$
H_{0}=\sum_{i=1}^{4} \frac{1}{2 m}\left(\frac{1}{i} \vec{\nabla}_{r_{i}}-q \vec{A}\left(r_{i}\right)\right)^{2},
$$

where $\vec{\nabla} \times \vec{A}=B$. Interactions (beyond the hardcore constraint) can be introduced by including a potential-energy term: $H=H_{0}+V\left(r_{1}, r_{2}, r_{3}, r_{4}\right)$.

It is worth mentioning that in general non-Abelian systems, the potential-energy term can be more complicated. Indeed, the most general potential-energy term for a fouranyon system is described not by a scalar but rather by a 2 $\times 2$ matrix $V_{\alpha \beta}\left(r_{1}, r_{2}, r_{3}, r_{4}\right)$, with $\alpha, \beta=1,2$. This $2 \times 2$ matrix is multivalued and transforms like $V_{\mathrm{ex}}=U^{-1} V U$ when two particles are exchanged. Physically, such a term describes an energy splitting between the two possible fusion outcomes of the four-particle system. In generic non-Abelian anyon systems, we expect such a splitting to be present although it will be exponentially small in the particle separation divided by a microscopic length scale (which is on the order of the magnetic length in the case of quantum Hall systems). Because of this exponential suppression and because of the complexity of including such terms, in this paper we will restrict our attention to scalar potential-energy interactions.

We can now easily generalize these results to a system of $2 N$ anyons. In the general case, the Hilbert space is described by row vectors $\left(\Psi_{1}, \ldots, \Psi_{2^{N-1}}\right)$ of $2^{N-1}$ functions of $2 N$ variables, which transform under appropriate unitary matrices when two particles are exchanged. We again impose the boundary condition that $\Psi$ vanishes as $r_{i} \rightarrow r_{j}$. As for the Hamiltonian, this is completely analogous to the four-anyon case.

\section{INCOMPRESSIBLE STATES OF NON-ABELIAN ANYONS IN A MAGNETIC FIELD}

In this section, we construct a series of incompressible states of non-Abelian anyons in a magnetic field. These 
states apply to a particular kind of anyon-anyons with the braiding statistics of the quasiholes in the Pfaffian state. (We will also take the anyons to be hardcore to avoid technical complications). We argue that these states are good candidate ground states in the case where the anyon interactions are short range, two-body, and repulsive, and the anyons are in their lowest effective Landau level.

We would like to mention that our model of short-range two-body repulsive interactions is motivated more by its simplicity than its realism. Indeed, in the physical case of the Pfaffian state at filling $\nu=5 / 2-\epsilon$, we expect that the quasihole interaction to be somewhat different. One can distinguish three parts to the interaction in this case. (1) There may be a long-range Coulomb interaction, falling off as $1 / r$, whose strength is determined by the quasiparticle charge $e^{*}$ and the dielectric constant of the material. In principle this piece could be absent, for example, if there is a nearby metallic gate which cuts off the Coulomb interaction at long distances. (2) At short distances there will be a portion of the interaction that we may describe by an effective potential, whose details are not known to us. (3) There will be interaction terms that depend on the fusion channel and cannot be represented by a simple position-dependent potential. As we discussed in Sec. II, these interactions have an energy scale $E \sim E_{0} \exp (-r / \xi)$ for some parameters $E_{0}, \xi$. The precise values of $E_{0}, \xi$ are unknown but $\xi$ is typically of the same order as the magnetic length $l^{*}$ for quasiparticles of charge $e^{*}$ $=e / 4$, while $E_{0}$ is of the same order as the energy gap $\Delta$, at least in some cases. In this paper, we ignore interactions of type (3), effectively considering the limit $\xi \ll l^{*}$ or $E_{0} \ll \Delta$. We also treat the rest of the interaction as a short-range repulsion. While neither of these assumptions is realistic, this model is at least a simple starting point.

We begin by analyzing the Landau-level physics of our non-Abelian anyons. Consider the Hamiltonian for $2 \mathrm{~N}$ noninteracting anyons with charge $q$ in a magnetic field $B$. In the general case where the anyons do not necessarily have quadratic dispersion, the appropriate Hamiltonian is of the form

$$
H_{0}=\sum_{i} F\left(\left[\frac{1}{i} \vec{\nabla}_{r_{i}}-q \vec{A}\left(r_{i}\right)\right]^{2}\right),
$$

where $F(x)$ is some increasing function for $x>0$, not necessarily linear, and $\vec{\nabla} \times \vec{A}=B$. Independent of the details of $F$, the lowest-energy states of this Hamiltonian are given by solutions $\left(\Psi_{1}, \ldots, \Psi_{2^{N-1}}\right)$ of

$$
\left(\frac{1}{i} \vec{\nabla}_{r_{i}}-q \vec{A}\left(r_{i}\right)\right)^{2} \Psi_{j}=|q| B \Psi_{j} .
$$

[This follows from the fact that the differential operator $\left(\frac{1}{i} \vec{\nabla}-q \vec{A}\right)^{2}$ has lowest eigenvalue $\left.|q| B\right]$. These low-energy states are highly degenerate, and all have energy $2 N F(|q| B)$. Physically, these states correspond to the case where all $2 \mathrm{~N}$ anyons are in their lowest effective Landau level. If the gap separating these lowest Landau-level states from higherenergy states is larger then the interaction energy scale, then we can restrict our Hilbert space to these states, replacing the anyon interaction with an appropriate lowest Landau-level interaction. Our problem then reduces to understanding which lowest Landau-level states are favored by the interactions. This is the limit we will consider here.

It is convenient to write down the lowest Landau-level states more explicitly. Let us focus on the case $q B<0$, and choose the circular gauge, $\vec{A}=B(x \hat{y}-y \hat{x}) / 2$. Using complex coordinates $w=x+i y$, the solutions to Eq. (3) can be written as

$$
\left(\widetilde{\Psi}_{1}\left(\left\{\bar{w}_{i}\right\}\right), \ldots, \widetilde{\Psi}_{2^{N-1}}\left(\left\{\bar{w}_{i}\right\}\right)\right) \exp \left(-\frac{1}{4 l^{2}} \sum_{i}\left|w_{i}\right|^{2}\right),
$$

where $\left(\widetilde{\Psi}_{1}, \ldots, \widetilde{\Psi}_{2^{N-1}}\right)$ are antianalytic in $w_{1}, \ldots, w_{2 N}$, and $l^{2}=1 /|q| B$. Since the particles in question are non-Abelian anyons, these functions need to be defined on an appropriate Riemann sheet and satisfy the transformation law described above under particle exchange.

We can now proceed to the problem of constructing candidate incompressible states. Following the logic of the Laughlin construction, we look for states which are (a) in the lowest Landau level and (b) have high-order zeros when two particles approach each other. Here, the second condition comes from our assumption that the anyons have short-range two-body repulsive interactions. Given the above parametrization of the lowest Landau level, this reduces to the problem of finding a row vector $\left(\widetilde{\Psi}_{1}, \ldots, \widetilde{\Psi}_{2^{N-1}}\right)$ of antianalytic functions satisfying the appropriate transformation law under particle exchange and having high-order zeros when $w_{i}$ $\rightarrow w_{j}$.

It is not obvious how to write down a collection of antianalytic functions with the appropriate transformation properties, much less one with high-order zeros when two coordinates coincide. However, the conformal field theory (CFT) ansatz for constructing trial fractional quantum Hall states provides a natural solution to both of these problems. The idea is to define a trial anyon wave function using a correlator from conformal field theory. ${ }^{1}$ In particular, consider the correlator

$\left\langle\bar{\sigma}\left(\bar{w}_{1}\right) \exp \left[i \sqrt{2 m+\frac{1}{8}} \bar{\phi}\left(\bar{w}_{1}\right)\right] \cdots \bar{\sigma}\left(\bar{w}_{2 N}\right) \exp \left[i \sqrt{2 m+\frac{1}{8}} \bar{\phi}\left(\bar{w}_{2 N}\right)\right]\right\rangle$

defined in a CFT which is a product of a chiral Ising theory ${ }^{1,17}$ and a chiral boson theory. Here, $m$ is a positive integer, $\bar{\sigma}$ is the spin operator in the chiral Ising theory, and $\bar{\phi}$ is a chiral boson operator in the chiral boson theory with normalization convention

$$
\begin{aligned}
\langle\exp & {\left.\left[i \sqrt{2 m+\frac{1}{8}} \bar{\phi}\left(\bar{w}_{1}\right)\right] \exp \left[-i \sqrt{2 m+\frac{1}{8}} \bar{\phi}\left(\bar{w}_{2}\right)\right]\right\rangle } \\
= & \left(\bar{w}_{1}-\bar{w}_{2}\right)^{-2 m-1 / 8} .
\end{aligned}
$$

This correlator has $2^{N-1}$ different conformal blocks-which we can label by $\alpha=1, \ldots, 2^{N-1}$. Thus, we can define $2^{N-1}$ antianalytic functions,

$$
\widetilde{\Psi}_{\alpha}^{m}\left(\bar{w}_{1}, \ldots, \bar{w}_{2 N}\right)=\left\langle\bar{\sigma}\left(\bar{w}_{1}\right) \exp \left[i \sqrt{2 m+1 / 8} \bar{\phi}\left(\bar{w}_{1}\right)\right] \cdots\right\rangle_{\alpha} .
$$

It is not hard to see that these anti-analytic functions satisfy the appropriate transformation law under particle exchange. Indeed, this follows from the monodromy properties of the above correlation function (e.g., its transformation law 
under analytic continuation). ${ }^{1,17}$ In addition, the correlation functions naturally satisfy the hardcore boundary condition that $\widetilde{\Psi} \rightarrow 0$ when $w_{i} \rightarrow w_{j}$. Thus, these states-which we will denote by $\Psi^{m}$-are legitimate many-body anyon states in the lowest Landau level.

For example, evaluating the correlator in the case $N=2$, one finds

$$
\widetilde{\Psi}_{1,2}^{m}\left(\bar{w}_{1}, \bar{w}_{2}, \bar{w}_{3}, \bar{w}_{4}\right)=\left(\sqrt{\bar{w}_{13} \bar{w}_{24}} \pm \sqrt{\bar{w}_{14} \bar{w}_{23}}\right)^{1 / 2} \prod_{i<j} \bar{w}_{i j}^{2 m},
$$

where $w_{i j}=w_{i}-w_{j}$. One can see that when particles 1 and 2 are exchanged in the counterclockwise direction, $\left(\widetilde{\Psi}_{1}, \widetilde{\Psi}_{2}\right)$ $\rightarrow\left(\widetilde{\Psi}_{1},-i \widetilde{\Psi}_{2}\right)$. This is exactly the unitary transformation we expect for a particle exchange. Moreover, one can see that $\widetilde{\Psi}_{1}, \widetilde{\Psi}_{2}$ vanish as $w_{i} \rightarrow w_{j}$.

In addition to being in the lowest Landau level, $\Psi^{m}$ also have the desired high-order zeros when particles approach each other. Indeed, one can see that $\Psi^{m}$ has a $2 m$ th order zero when $w_{i} \rightarrow w_{j}$. (More specifically, $\Psi^{m}$ vanishes like $\left(\bar{w}_{i}-\bar{w}_{j}\right)^{2 m}$ when $i, j$ are in the identity fusion channel, and $\left(\bar{w}_{i}-\bar{w}_{j}\right)^{2 m+1 / 2}$ when $i, j$ are in the $\psi$ fusion channel.)

Since $\Psi^{m}$ have the desired properties (a) and (b), they appear to be good candidate ground states for short-range two-body repulsive interactions. However, to complete the story, it would be good to find a specific interaction for which they are the exact ground states. Such a model interaction is easy to construct. Indeed, let $V=V(r / a)$ be a shortrange repulsive two-body interaction with range $a$. The model interaction we consider is simply $V$ in the limit of small $a$ (e.g., $a \ll l$ ). (Note that this is the same as the model interaction which stabilizes the Laughlin states.)

To see why $\Psi^{m}$ is the ground state for this interaction, note that the interaction energy of $\Psi^{m}$ is very small for small $a$ due to the presence of the $2 m$ th order zeros:

$$
\left\langle\Psi^{m}\left|\sum_{i<j} V\left(\boldsymbol{r}_{i}-\boldsymbol{r}_{j}\right)\right| \Psi^{m}\right\rangle \propto\left(\frac{a}{l}\right)^{4 m+2} .
$$

Also, it is not hard to see that $\Psi^{m}$ scales like $w_{i}^{2 m(2 N-1)+(N-1) / 4}$ as $w_{i} \rightarrow \infty$. This means that the highest single-particle angular momentum in $\Psi^{m}$ is $L_{\max }=2 m(2 N-1)+(N-1) / 4$. We believe that any other state with maximum angular momentum $L_{\max }$ has at least one lower-order zero. We have not proven this statement, but it appears to hold for small systems. For example, in the $N=2$ case, one can prove that $\Psi^{m}$ is the unique state with maximum angular momentum $L_{\max }=6 \mathrm{~m}$ $+1 / 4$ and $2 m$ th order zeros. Assuming this is true is general, it follows that all other states on an appropriately sized disk or sphere have an interaction energy which goes to zero slower than $(a / l)^{4 m+2}$. Thus, in the limit $a \rightarrow 0, \Psi^{m}$ will be the unique ground state.

We cannot make any analytic arguments for the existence of a finite gap. This must be checked numerically. However, given the analogy to the Laughlin states, a gap seems likely for small $m$. On the other hand, when $m$ is large, the system may be gapless since in that case $\Psi^{m}$ may describe a Wigner crystal rather than a uniform density liquid.
Assuming that $\Psi^{m}$ is gapped for small $m$, it is necessarily translationally invariant and thus an incompressible liquid. We have therefore accomplished our goal of constructing incompressible liquid states of non-Abelian anyons in a magnetic field. The filling fractions of these states are given by

$$
\nu_{\text {anyon }}=\lim _{N \rightarrow \infty} \frac{2 N}{L_{\max }}=\frac{1}{2 m+1 / 8} .
$$

\section{PROPERTIES OF THE HIERARCHICAL STATES}

We now return to the physics of the Pfaffian state away from half filling. We investigate the hierarchical quantum Hall states $\Psi_{h i}^{m}$ that would result if the quasiholes formed one of the $\Psi^{m}$ states.

To begin, assume we have a FQH system whose ground state at half filling is the Pfaffian state and suppose we increase the magnetic field so that the system nucleates a finite density of quasiholes. Since these quasiholes feel a magnetic field with $e^{*} B<0$, and carry the appropriate non-Abelian statistics, they could in principle form one of the incompressible liquids described by $\Psi^{m}$.

In practice, these states may not be realized. As we mentioned earlier, our model of pointlike non-Abelian anyons with short-range two-body repulsive interactions is not a good description of a realistic FQH system - especially when the quasihole density is high. On the other hand, when the quasihole density is very low, it is likely that the quasiholes will form a Wigner crystal. Nevertheless, it is still interesting conceptually to understand the properties of these hypothetical hierarchical states $\Psi_{\text {hi }}^{m}$.

First, let us compute the filling fraction for these states. Recall that the anyon filling fraction in $\Psi^{m}$ is $\nu_{\text {anyon }}$ $=1 /(2 m+1 / 8)$. Since the anyons in this case are quasiholes with charge $e / 4$, the corresponding electron filling fraction in $\Psi_{\mathrm{hi}}^{m}$ is

$$
\nu=\frac{1}{2}-\frac{1}{16} \nu_{\text {anyon }}=\frac{8 m}{16 m+1} .
$$

In particular, the simplest hierarchical state (with $m=1$ ) occurs at $\nu=8 / 17$.

Next, we write down wave functions for these states in terms of the original electron coordinates $\{z\} \equiv\left\{z_{1}, \ldots, z_{2 M}\right\}$. The logic is similar to the usual Abelian hierarchical construction but it is worth repeating here, given the unfamiliar context. In Sec. III, we thought of the wave function $\Psi_{\alpha}^{m}(\{\bar{w}\})$ as defining a state in a Hilbert space for pointlike non-Abelian anyons. This state was given by

$$
\left|\Psi^{m}\right\rangle=\int d w \sum_{\alpha} \Psi_{\alpha}^{m}(\{\bar{w}\}) \cdot|\{w\}, \alpha\rangle,
$$

where $|\{w\}, \alpha\rangle$ denotes the state in which the non-Abelian anyons are at positions $\{w\}$ and in fusion state $\alpha$. Now we consider the case where the non-Abelian anyons are actually quasihole excitations in the Pfaffian state. In this context, the Hilbert space for non-Abelian anyons is actually a subspace of the full electron Hilbert space-the subspace spanned by 
$2 N$ quasihole states. Each state $|\{w\}, \alpha\rangle$ corresponds to some linear combination of electron basis states $|\{z\}\rangle$ :

$$
|\{w\}, \alpha\rangle=\int d z \Phi_{\alpha, q h}(\{z\} ;\{w\}) \cdot|\{z\}\rangle .
$$

The coefficient in this expansion, $\Phi_{\alpha, \mathrm{qh}}$, is the electron wave function for fixed quasihole positions $\{w\}$ and fusion state $\alpha$.

Combining Eqs. (11) and (12), we see that $\left|\Psi^{m}\right\rangle$ corresponds to the hierarchical state

$$
\left|\Psi_{\mathrm{hi}}^{m}\right\rangle=\int d w d z \sum_{\alpha} \Psi_{\alpha}^{m}(\{\bar{w}\}) \Phi_{\alpha, \mathrm{qh}}(\{z\} ;\{w\}) \cdot|\{z\}\rangle .
$$

Equivalently, in wave-function notation,

$$
\Psi_{\mathrm{hi}}^{m}(\{z\})=\int d w \sum_{\alpha} \Psi_{\alpha}^{m}(\{\bar{w}\}) \Phi_{\alpha, \mathrm{qh}}(\{z\} ;\{w\}) .
$$

To proceed further, we use the explicit form for the quasihole wave functions $\Phi_{\alpha, q h}$. Like $\Psi^{m}$, they can be written as $\Phi_{\alpha, \mathrm{qh}}=\widetilde{\Phi}_{\alpha, \mathrm{qh}} \cdot \exp \left(-1 / 4 l^{2} \Sigma_{i}\left|z_{i}\right|^{2}\right)$, where $\widetilde{\Phi}_{\alpha, \mathrm{qh}}$ is a CFT correlator. ${ }^{1}$ Specifically,

$$
\widetilde{\Phi}_{\alpha, \mathrm{qh}}(\{z\} ;\{w\})=\left\langle\sigma\left(w_{1}\right) e^{i / \sqrt{8} \phi\left(w_{1}\right)} \cdots \psi\left(z_{1}\right) \exp \left[i \sqrt{2} \phi\left(z_{1}\right)\right] \cdots\right\rangle_{\alpha} .
$$

Combining the two correlators into one, the sum in Eq. (14) can be simplified to

$$
\begin{aligned}
& \sum_{\alpha} \Psi_{\alpha}^{m}(\{\bar{w}\}) \Phi_{\alpha, \mathrm{qh}}(\{z\} ;\{w\}) \\
&=\left\langle\sigma\left(w_{1}, \bar{w}_{1}\right) \exp \left[\frac{i}{\sqrt{8}} \phi\left(w_{1}\right)+i \sqrt{2 m+\frac{1}{8}} \bar{\phi}\left(\bar{w}_{1}\right)\right]\right. \\
&\left.\cdots \psi\left(z_{1}\right) \exp \left[i \sqrt{2} \phi\left(z_{1}\right)\right] \cdots\right\rangle \\
& \times \exp \left(-\frac{1}{4 l^{2}} \sum_{i}\left|z_{i}\right|^{2}-\frac{1}{16 l^{2}} \sum_{i}\left|w_{i}\right|^{2}\right),
\end{aligned}
$$

where $\sigma(w, \bar{w})$ is the spin field in the nonchiral Ising model. ${ }^{17}$

This simplification is useful because it expresses the wave function $\Psi_{\mathrm{hi}}^{m}$ in terms of a single CFT correlator and thus allows us to use conformal field theory to analyze the state. ${ }^{1}$ This conformal field-theory approach is very powerful and allows us to quickly extract the universal properties of the quasiparticles and edge excitations. However, it is worth mentioning that the CFT approach is more a series of conjectures then a rigorous method. It is known to work in some cases (such as the Laughlin state and Moore-Read state ${ }^{18}$ ) and is believed to work in many others, but it has not been proven in generality. Therefore, the results below-like the existence of a gap-need independent (numerical) verification.

We begin with an analysis of the quasiparticle spectrum. According to the conformal field-theory approach, quasiparticle excitations can be constructed by inserting operators $\mathcal{O}\left(z_{0}\right)$ into the above correlation function [Eq. (16)]. The operators $\mathcal{O}$ can be arbitrary except that they must be local with respect to the electron operator $\psi(z) e^{i \sqrt{2} \phi(z)}$ and the quasihole operator $\sigma(z, \bar{z}) e^{i[\sqrt{1 / 8} \phi(z)+\sqrt{2 m+1 / 8} \bar{\phi}(\bar{z})]}$-that is, their correlation functions with these operators must be single valued. (This requirement comes from the fact that the excited state wave function must be single valued in the electron and quasihole coordinates). The second part of the conformal field-theory conjectures is that, in the thermodynamic limit, the Berry phase associated with exchanging two quasiparticle excitations with coordinates $z_{0}, z_{1}$ is exactly given by the monodromy of the associated correlation function $\left\langle\mathcal{O}\left(z_{0}\right) \mathcal{O}\left(z_{1}\right) \ldots\right\rangle$ under such an exchange (e.g., the phase associated with analytically continuing the correlation function along a path exchanging $\left.z_{0}, z_{1}\right)$. This is known to be correct in the Laughlin case-where one can explicitly calculate the Berry phase using the plasma analogy - but it has not been established in more complicated cases such as the one in question.

Assuming that these conjectures can be applied in this case, we simply need to look for operators $\mathcal{O}$ which are local with respect to the electron and quasihole operator. We can then define an equivalence relation on these operators by setting $\mathcal{O}_{1} \equiv \mathcal{O}_{2}$ if correlation functions involving $\mathcal{O}_{1} \mathcal{O}_{2}^{-1}$ and any other allowed operators are single valued. The resulting equivalence classes should then be in one-to-one correspondence with topologically distinct quasiparticle excitations.

We begin with the set of operators of the form $\left\{\chi e^{i a \phi+i b \bar{\phi}}\right\}$, where $\chi=1, \psi, \sigma, \mu$, and $a, b$ are real numbers (in principle, one should consider even more general operators with spatial derivatives, etc., but these do not appear to give new equivalence classes). Imposing locality with respect to the electron and quasihole operator, we find the following list of allowed operators, or more accurately, equivalence classes of operators: $\left\{\chi e^{i\left(n_{1} \phi / \sqrt{2}+\left(n_{2}+n_{1} / 4\right) \bar{\phi} / \sqrt{2 m+1 / 8}\right)}\right\}$, where $n_{1}$ is an integer or half integer depending on whether $\chi=1, \psi$ or $\chi=\sigma, \mu$ and $n_{2}$ is integer or half-integer depending on whether $\chi=1, \sigma$ or $\chi=\psi, \mu$. One can check that all of these operators can be generated from the operator $\psi e^{i \bar{\phi} / 2 \sqrt{2 m+1 / 8}}$ (and electron and quasihole operators). We conclude that all of the (topologically distinct) quasiparticle excitations are composites of the elementary quasiparticle corresponding to $\mathcal{O}=\psi e^{i \bar{\phi} / 2 \sqrt{2 m+1 / 8}}$.

The charge of this excitation can be computed in many ways. One way, which does not involve too much formalism, is to consider $\mathcal{O}^{2}=e^{i \bar{\phi} / \sqrt{2 m+1 / 8}}$. An insertion of $\mathcal{O}^{2}\left(w_{0}\right)$ into correlation function (16) gives the same correlation function back but with an additional multiplicative factor of $\Pi_{i}\left(w_{i}\right.$ $\left.-w_{0}\right)$. Thus this operator creates a Laughlin-type quasihole in the quasihole condensate. Since the quasiholes are at filling $\nu_{\text {anyon }}=1 /(2 m+1 / 8)$ and the quasiholes carry charge $e / 4$, the charge accumulated at $w_{0}$ is

$$
\frac{e}{4} \frac{1}{2 m+1 / 8}=\frac{2 e}{16 m+1} .
$$

Dividing this result by 2, we conclude that the charge associated with the elementary quasiparticle/quasihole is $\pm e /(16 m+1)$.

As for the statistics, note that the two point correlator for $\mathcal{O}$ is $\left\langle\mathcal{O}\left(w_{1}\right) \mathcal{O}\left(w_{2}\right)\right\rangle=w_{12}^{-1} \bar{w}_{12}^{2 /(16 m+1)}$. The phase accumulated by this correlation function under a counterclockwise ex- 
change of $w_{1}, w_{2}$ is therefore $e^{i \theta}=e^{i \pi(16 m-1) /(16 m+1)}$. Applying the conformal field-theory conjectures, this is precisely the statistical (Berry) phase associated with a counterclockwise quasiparticle exchange. Note, in particular, that the elementary quasiparticle is an Abelian anyon. This means that all the quasiparticles are Abelian anyons (since they can be generated as composites of the elementary quasiparticle). Thus, the hierarchical state $\Psi_{\text {hi }}^{m}$ is actually an Abelian quantum Hall state-even though the parent Pfaffian state is non-Abelian.

This result is not as strange as it first appears. Indeed, as we mentioned earlier, previous analysis ${ }^{13}$ has shown that an array of pinned quasiholes (or equivalently a quasihole Wigner crystal) can give rise to an Abelian state (see also Ref. 14 and in the disordered case, Ref. 15). It is therefore not surprising that a finite density of free quasiholes can give rise to Abelian (hierarchical) states.

To complete our analysis of $\Psi_{\text {hi }}^{m}$, we compute its thermal Hall conductance $K_{H}$. Our computation is based on the general correspondence between the edge modes of a quantum Hall state and the modes of the conformal field theory used to define its wave function. ${ }^{19}$ Applying this to $\Psi_{\mathrm{hi}}^{m}$, we see that the edge contains one forward propagating Majorana mode [corresponding to $\psi(z)$ ], one backward propagating Majorana mode [corresponding to $\bar{\psi}(\bar{z})$ ], one forward propagating boson [corresponding to $\phi(z)]$, and one backward propagating boson [corresponding to $\bar{\phi}(\bar{z})]$. Recall that each chiral boson mode gives a contribution of $\pm \frac{\pi^{2} k_{B}^{2}}{3 h} T$ to the thermal Hall conductance, while each Majorana mode contributes half as much. The total thermal Hall conductance for $\Psi_{\mathrm{hi}}^{m}$ is therefore $(1 / 2-1 / 2+1-1) \frac{\pi^{2} k_{B}^{2}}{3 h} T=0$.

\section{RELATION TO THE JAIN STATES AND THE STRONG PAIRING STATE}

Since $\Psi_{\mathrm{hi}}^{m}$ is Abelian, it is natural to wonder how it relates to the usual Jain state (or equivalently, hierarchical state ${ }^{10}$ ) at filling $\nu=8 m /(16 m+1)$. To this end, note that the elementary quasiparticle/quasihole in the Jain state has charge $\pm e /(16 m+1)$ and statistical phase $e^{i \pi(16 m-1) /(16 m+1)}$. Similarly, note that the thermal Hall conductance of the Jain state is $8 m$ (in units of $\frac{\pi^{2} k_{B}^{2}}{3 h} T$ ). Comparing with the results above, we see that while the quasiparticle charges and statistics of the two states are identical, their thermal Hall conductances are different. Thus $\Psi_{\text {hi }}^{m}$ is distinct from the $\nu=8 m /(16 m+1)$ Jain state.

A simpler, but less fundamental distinction between the two states can be obtained by examining their shifts $\mathcal{S}$ on the sphere. One can check that for the Jain state, the number of flux quanta $N_{\phi}$ is related to the number of electrons $N_{e}$ by

$$
N_{\phi}=\frac{16 m+1}{8 m} N_{e}-(8 m+2) \text {. }
$$

Thus the shift is $\mathcal{S}=8 m+2$. On the other hand, a simple calculation shows that the shift for $\Psi_{\mathrm{hi}}^{m}$ is $\mathcal{S}=5 / 2$. [One way to derive this is to note that the integral in Eq. (14) is only nonvanishing on the sphere if each quasihole coordinate $w_{i}$ occurs with the same maximum power as $\bar{w}_{i}$. This leads to the relation $N_{e} / 2=2 m\left(N_{\mathrm{qh}}-1\right)$ where $N_{e}, N_{\mathrm{qh}}$ are the number of electrons and quasiholes, respectively. Combining this with the relation between the number of flux quanta and number of quasiholes in the Pfaffian state, $N_{\phi}=2 N_{e}-3$ $+N_{\mathrm{qh}} / 2$, gives the required shift $\left.\mathcal{S}=5 / 2\right]$. This difference in shifts provides a simple way to distinguish the two states numerically.

While the $\Psi_{\mathrm{hi}}^{m}$ are distinct from the Jain states and the standard hierarchical states, we would like to mention that they appear to be equivalent to hierarchical states derived from the strong-pairing $\nu=1 / 2$ state. ${ }^{11}$ Indeed, consider the strong-pairing $\nu=1 / 2$ state, which can be thought of as a $\nu$ $=1 / 8$ Laughlin state of tightly bound pairs of electrons. The elementary quasihole in this state carries charge $e / 4$ and statistical phase $e^{i \pi / 8}$. Following the usual Abelian hierarchy construction, ${ }^{5,6}$ one can consider Laughlin-type incompressible liquids formed out of these quasiholes. These states can occur at quasihole filling fractions $\nu_{\mathrm{qh}}=1 /(2 m+1 / 8)$ for any positive integer $m$. The corresponding electron filling fraction for these states is given by

$$
\nu=\frac{1}{2}-\frac{1}{16} \nu_{\mathrm{qh}}=\frac{8 m}{16 m+1} .
$$

Thus, we see that these states occur at the same filling fraction at $\Psi_{\text {hi }}^{m}$. In addition, one can check that the elementary quasiparticle/quasihole has charge $\pm e /(16 m+1)$ and statistical phase $e^{i \pi(16 m-1) /(16 m+1)}$. Finally, it is easy to see that the thermal Hall conductance of this state vanishes. All of these properties agree exactly with the $\Psi_{\mathrm{hi}}^{m}$. Therefore, it appears that they carry the same topological order. We expect that they are in the same universality class-that is, one can go from one state to the other by continuously varying parameters in the Hamiltonian, without a phase transition.

\section{QUASIPARTICLES VS QUASIHOLES}

So far we have focused our attention on finding incompressible states formed out of quasiholes. However, it is equally natural to consider states formed out of quasiparticles. In this section, we address this question. We construct a series of incompressible hierarchical states $\chi_{\mathrm{hi}}^{m}$, analogous to $\Psi_{\text {hi }}^{m}$ but composed out of quasiparticles.

The first step is to construct incompressible states of pointlike non-Abelian anyons in a strong magnetic field. These anyons should carry the statistics and charges of the quasiparticles in the Pfaffian state.

Our approach, as before, is to construct lowest Landaulevel states with high-order zeros when particles coincide. Since quasiparticles carry the opposite charge of quasiholes, these lowest Landau-level wave functions are given by row vectors $\left(\widetilde{\chi}_{1}, \ldots, \widetilde{\chi}_{2} N-1\right)$ of $2^{N-1}$ analytic functions rather than antianalytic functions. On the other hand, since quasiparticles have the same statistics as quasiholes, the $\tilde{\chi}_{i}$ must transform in the same way as before under particle exchange.

Previously, we constructed a series of lowest Landaulevel states with the appropriate zero structure using the conformal blocks of a CFT correlator 


$$
\left\langle\bar{\sigma}\left(\bar{w}_{1}\right) \exp \left[i \sqrt{2 m+\frac{1}{8}} \bar{\phi}\left(\bar{w}_{1}\right)\right] \cdots \bar{\sigma}\left(\bar{w}_{2 N}\right) \exp \left[i \sqrt{2 m+\frac{1}{8}} \bar{\phi}\left(\bar{w}_{2 N}\right)\right]\right\rangle_{\alpha} .
$$

Since in this case we need analytic functions rather than anti-analytic functions, it is tempting to use the same approach but with the correlator

$$
\left\langle\sigma\left(w_{1}\right) \exp \left[i \sqrt{2 m+\frac{1}{8}} \phi\left(w_{1}\right)\right] \cdots \sigma\left(w_{2 N}\right) \exp \left[i \sqrt{2 m+\frac{1}{8}} \phi\left(w_{2 N}\right)\right]\right\rangle_{\alpha} .
$$

Unfortunately, this construction does not work as the resulting collection of analytic functions $\widetilde{\chi}_{\alpha}^{m}$ satisfy the opposite transformation law from what is required. That is, they transform as $\widetilde{\chi}_{\alpha, \text { ex }}^{m}=\Sigma_{\beta} \widetilde{\chi}_{\beta}^{m} U_{\beta \alpha}$ instead of $\widetilde{\chi}_{\alpha, \text { ex }}^{m}=\Sigma_{\beta} \widetilde{\chi}_{\beta}^{m} U_{\beta \alpha}^{*}$ (here $U_{\alpha \beta}$ is the unitary matrix associated with the particle exchange).

To construct a legitimate state, we need to modify the correlator so that it has the opposite monodromy under particle exchange. To this end, we separate out the Abelian part of the correlator and then regroup terms

$$
\begin{aligned}
& \left\langle\sigma\left(w_{1}\right) \exp \left[i \sqrt{2 m+\frac{1}{8}} \phi\left(w_{1}\right)\right] \cdots\right\rangle_{\alpha} \\
& =\prod_{i<j} w_{i j}^{2 m+1 / 8}\left\langle\sigma\left(w_{1}\right) \cdots\right\rangle_{\alpha} \\
& =\left(\prod_{i<j} w_{i j}^{-1 / 8}\left\langle\sigma\left(w_{1}\right) \cdots\right\rangle_{\alpha}\right)\left(\prod_{i<j} w_{i j}^{2 m+1 / 4}\right) .
\end{aligned}
$$

We can reverse the monodromy of the second term by simply changing the exponent $2 m+1 / 4 \rightarrow 2 m-1 / 4$. Reversing the monodromy of the first term is more complicated. However, we can achieve this goal using the fact that the monodromy of this term is described by the spinor representation of $\mathrm{SO}(2 N)$. Indeed, this term transforms under a particle exchange $i \leftrightarrow j$, just like the $2^{N-1}$ basis vectors of the spinor representation transform under a $\pi / 2$ rotation in the $i j$ plane. ${ }^{20}$ Since the spinor representation is equivalent to its conjugate when $N$ is even, one can obtain an expression with the opposite monodromy by simply multiplying by the (unitary) matrix $R_{\alpha \beta}$ which describes this equivalence. In more detail: let $R_{\alpha \beta}$ be the unique (up to phase) unitary matrix with the property that $R_{\alpha \beta}^{-1} U_{\beta \gamma} R_{\gamma \delta}=U_{\alpha \delta}^{*}$ for all the unitary matrices $U$ in the spinor representation of $\mathrm{SO}(2 N)$. Then, $\Pi_{i<j} w_{i j}^{-1 / 8}\left\langle\sigma\left(w_{1}\right) \cdots\right\rangle_{\beta} R_{\beta \alpha}$ has the opposite monodromy from $\Pi_{i<j} w_{i j}^{-1 / 8}\left\langle\sigma\left(w_{1}\right) \cdots\right\rangle_{\alpha}$ under particle exchange. One can give an explicit form for $R$ if one labels the conformal blocks by $\alpha=\left(\alpha_{1}, \ldots, \alpha_{N-1}\right)$, where $\alpha_{i}=1,2$ depending on whether particles $2 i-1,2 i$ are in the $1, \psi$ fusion channel. In that basis, $R$ can be written as a product of Pauli matrices, $R$ $=\sigma_{1}^{y} \sigma_{2}^{x} \sigma_{3}^{y} \sigma_{4}^{x} \cdots \sigma_{N-1}^{y} \cdot{ }^{21}$

Making these two modifications and recombining the two terms, we arrive at the following candidate states:

$$
\tilde{\chi}_{\alpha}^{m}\left(w_{1}, \ldots, w_{2 N}\right)=\left\langle\sigma\left(w_{1}\right) \exp \left[i \sqrt{2 m-\frac{3}{8}} \phi\left(w_{1}\right)\right] \ldots\right\rangle_{\beta} R_{\beta \alpha} .
$$

By construction, the $\widetilde{\chi}_{\alpha}^{m}$ are analytic and transform in the appropriate way under particle exchange. Thus, they describe legitimate lowest Landau-level states.

As an example, consider the case $N=2$. In that case, the $\tilde{\chi}_{\alpha}^{m}$ are given (up to constant factor) by

$$
\widetilde{\chi}_{1,2}^{m}\left(w_{1}, w_{2}, w_{3}, w_{4}\right)= \pm\left(\sqrt{w_{13} w_{24}} \mp \sqrt{w_{14} w_{23}}\right)^{1 / 2} \prod_{i<j} w_{i j}^{2 m-1 / 2}
$$

where $w_{i j}=w_{i}-w_{j}$. One can see that when particles 1 and 2 are exchanged, $\left(\tilde{\chi}_{1}, \tilde{\chi}_{2}\right) \rightarrow\left(\tilde{\chi}_{1},-i \tilde{\chi}_{2}\right)$. This is exactly the required unitary transformation.

In addition to being in the lowest Landau level, the $\chi^{m}$ satisfy our condition of having high-order zeros when two particles approach each other. Thus, they appear to be good candidate ground states for a model with short-range twobody repulsive interactions. As before, one can complete the picture by constructing model interactions for which they are the exact ground state.

Thus, just like the $\Psi^{m}$, the $\chi^{m}$ suggest a series of hierarchical states $\chi_{h i}^{m}$ that can arise from the Pfaffian state. These states will occur if there is a finite density of quasiparticles, and the quasiparticles form one of the $\chi^{m}$ states.

What are the properties of these states? Let us begin with the filling fraction. Since the anyon filling fraction for $\chi^{m}$ is $\nu_{\text {anyon }}=1 /(2 m-3 / 8)$, the electron filling fraction for $\chi_{h i}^{m}$ is

$$
\nu=\frac{1}{2}+\frac{1}{16} \nu_{\text {anyon }}=\frac{8 m-1}{16 m-3} .
$$

In particular, the simplest hierarchical state (corresponding to $m=1$ ) occurs at $\nu=7 / 13$.

Next, we write down the wave functions for these states in terms of the electron coordinates, $\{z\}=\left\{z_{1}, \ldots, z_{2 M}\right\}$. We have

$$
\chi_{h i}^{m}(\{z\})=\int d w \sum_{\alpha} \chi_{\alpha}^{m}(\{w\}) \Phi_{\alpha, \mathrm{qp}}(\{z\} ;\{w\}),
$$

where $\chi_{\alpha}^{m}=\tilde{\chi}_{\alpha}^{m} \cdot \exp \left[1 / 16 l^{2} \Sigma_{i}\left|w_{i}\right|^{2}\right]$ are the quasiparticle wave functions, and $\Phi_{\alpha, \mathrm{qp}}=\tilde{\Phi}_{\alpha, \mathrm{qp}} \cdot \exp \left[-1 / 4 l^{2} \Sigma_{i}\left|z_{i}\right|^{2}\right]$ are the electron wave functions for fixed quasiparticle positions $\{w\}$.

Unlike the quasihole case, there is no canonical form for these quasiparticle wave functions. We will use the wave function

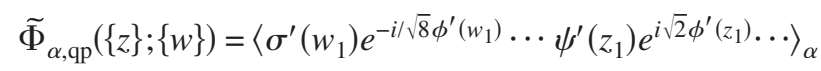

as it is particularly convenient for our analysis. (Note that this wave function has unphysical singularities as $w_{i} \rightarrow z_{j}$. Strictly speaking these singularities need to be regularized in some way. However, we will ignore this regularization as it plays no role in the universal long-distance structure of the wave function.)

We next make use of the identity

$$
\begin{aligned}
& \left\langle\sigma\left(w_{1}\right) \cdots\right\rangle_{\alpha}\left\langle\sigma^{\prime}\left(w_{1}\right) \cdots \psi^{\prime}\left(z_{1}\right) \cdots\right\rangle_{\beta} R_{\alpha \beta} \\
& =\left\langle e^{i / 2 \phi^{\prime \prime}\left(w_{1}\right)} \cdots \cos \left[\phi^{\prime \prime}\left(z_{1}\right)\right] \cdots\right\rangle
\end{aligned}
$$

(up to a constant factor), where $\phi^{\prime \prime}$ is a free boson field. [A formal justification of this identity can be found in (Ref. 22) in the second paragraph after Eq. (7.32). In addition, we have explicitly verified the relation for $N=0,1,2$.] Applying this identity, the sum in Eq. (23) can be simplified to 


$$
\begin{aligned}
& \sum_{\alpha} \chi_{\alpha}^{m}(\{w\}) \Phi_{\alpha, \mathrm{qp}}(\{z\} ;\{w\}) \\
&=\left\langle\exp \left[i \sqrt{2 m-\frac{3}{8}} \phi\left(w_{1}\right)-\frac{i}{\sqrt{8}} \phi^{\prime}\left(w_{1}\right)+\frac{i}{2} \phi^{\prime \prime}\left(w_{1}\right)\right]\right. \\
&\left.\cdots e^{i \sqrt{2} \phi^{\prime}\left(z_{1}\right)} \cos \left(\phi^{\prime \prime}\left(z_{1}\right)\right) \cdots\right\rangle \\
& \times \exp \left(-\frac{1}{4 l^{2}} \sum_{i}\left|z_{i}\right|^{2}-\frac{1}{16 l^{2}} \sum_{i}\left|w_{i}\right|^{2}\right)
\end{aligned}
$$

We have now expressed $\chi_{\text {hi }}^{m}$ in terms of a single CFT correlator and are therefore in a position to use the CFT approach to compute its properties. A calculation analogous to the one for $\Psi_{\mathrm{hi}}^{m}$ shows that all the quasiparticle excitations are composites of the elementary quasiparticle corresponding to $\mathcal{O}=e^{i \phi / 2 \sqrt{2 m-3 / 8}-i \phi^{\prime \prime}}$. It is also straightforward to show that the elementary quasiparticle/quasihole carries charge $\pm e /(16 m-3)$ and exchange statistics $e^{i \theta}=e^{i \pi(16 m-1) /(16 m-3)}$. Thus, $\chi_{h i}^{m}$ is an Abelian state-just like $\Psi_{h i}^{m}$. As for the thermal Hall conductance, note that the edge contains three forward propagating chiral bosons [corresponding to $\phi, \phi^{\prime}, \phi^{\prime \prime}$ so that the total thermal Hall conductance is $1+1+1=3$ (in units of $\left.\frac{\pi^{2} k_{B}^{2}}{3 h} T\right)$ ].

How does this state relate to the Jain state at filling $\nu$ $=(8 m-1) /(16 m-3)$ ? A simple calculation shows that the elementary quasiparticle in the Jain state has the same charge and statistics as $\chi_{\mathrm{hi}}^{m}$. Nevertheless the two states are distinct as they have different thermal Hall conductances: the thermal Hall conductance for the Jain state is $3-8 m$ (in appropriate units) - rather than 3 . In addition, while the shift for the Jain state is $\mathcal{S}=3-8 m$, the shift for $\chi_{\text {hi }}^{m}$ is $\mathcal{S}=(28 m-3) /(8 m-1)$. This difference in shifts provides a simple way to distinguish the two states numerically.

While the $\chi_{\text {hi }}^{m}$ are distinct from the Jain states, they appear to be equivalent to hierarchical states derived from the 331 $\nu=1 / 2$ state. ${ }^{11,12}$ Indeed, the elementary quasiparticle in the 331 state carries charge $e / 4$ and statistical phase $e^{3 i \pi / 8}$. Following the usual hierarchical construction, ${ }^{5,6}$ these quasiholes can form Laughlin-type incompressible liquids at quasiparticle filling fractions $\nu_{\mathrm{qp}}=1 /(2 m-3 / 8)$. The corresponding electron filling fraction for these states is

$$
\nu=\frac{1}{2}+\frac{1}{16} \nu_{\mathrm{qp}}=\frac{8 m-1}{16 m-3} .
$$

Thus, these states occur at the same filling fraction as $\chi_{\mathrm{hi}}^{m}$. In addition, one can check that the elementary quasiparticle/ quasihole has charge $\pm e /(16 m-3)$ and statistical phase $e^{i \pi(16 m-1) /(16 m-3)}$. Finally, it is easy to see that the thermal Hall conductance of this state is 3 in appropriate units. All of these properties agree exactly with the $\chi_{\mathrm{hi}}^{m}$. Therefore, it appears that they carry the same topological order. We expect that they are in the same universality class - that is, one can go from one state to the other by continuously varying parameters in the Hamiltonian, without a phase transition.

\section{HIGHER LEVELS IN THE HIERARCHY}

As in the original Abelian hierarchy, the states $\Psi_{\mathrm{hi}}^{m}$ and $\chi_{\mathrm{hi}}^{m}$ may give rise to daughter states with filling fractions near $8 m /(16 m+1)$ and $(8 m-1) /(16 m-3)$ respectively. These second level hierarchical states may then give rise to their own daughter states and so on. In this way, the Pfaffian state may give rise to an infinite hierarchy of quantum Hall states. We will not describe this hierarchy in detail, since the parent states $\Psi_{\mathrm{hi}}^{m}$ and $\chi_{\mathrm{hi}}^{m}$ are Abelian and therefore the analysis is very similar to the original Abelian hierarchy. ${ }^{5,6}$ Nevertheless, with very little work, we can say quite a bit about these higher level hierarchical states.

The key point is that $\Psi_{\mathrm{hi}}^{m}$ and $\chi_{\mathrm{hi}}^{m}$ have the same quasiparticle charges and statistics as the usual Abelian hierarchical states at the corresponding filling fraction. Since these quantities are the only ones that enter in the hierarchical construction, it follows that the descendant states of $\Psi_{\mathrm{hi}}^{m}$ and $\chi_{\mathrm{hi}}^{m}$ must also have the same filling fractions, quasiparticle charges, and quasiparticle statistics as the usual Abelian hierarchical states. (Despite this similarity, these states are distinct from the usual Abelian hierarchical states: just as $\Psi_{\mathrm{hi}}^{m}$ and $\chi_{\mathrm{hi}}^{m}$ have a different thermal Hall conductance from the corresponding Abelian hierarchical states, this will also hold for all descendant states.)

This result allows us to compute the universal properties of the higher level states very easily. In particular, we can find the filling fractions for these states. One finds that the hierarchy gives rise to all odd denominator fractions in the range $15 / 32<\nu<13 / 24$ and no others. (Here, we have assumed, as in the original hierarchy construction that the descendant states of $\Psi_{\mathrm{hi}}^{m}$ and $\chi_{\mathrm{hi}}^{m}$ are constructed out of Laughlin-type states of elementary quasiparticles or quasiholes - that is quasiparticles or quasiholes with the minimal charge. If instead, the quasiparticles cluster into higher charges which then form Laughlin-type states, other filling fractions can be realized.) We would like to mention though that unlike the usual hierarchy, one can obtain multiple states at the same filling fraction. For example, two $\nu$ $=16 / 33$ states can be obtained: one as the $\Psi_{h i}^{2}$ state and one as a descendant of the $8 / 17$ state. These two $\nu=16 / 33$ states have the same quasiparticle statistics and charges but different thermal Hall conductances.

\section{ANTIPFAFFIAN VS PFAFFIAN}

The discussion so far has focused on hierarchical states derived from the Pfaffian states. However, since the particlehole conjugate of the Pfaffian (or "anti-Pfaffian",3) is an equally strong candidate for the observed $\nu=5 / 2$ plateau, it is worth applying the analysis to this state as well.

The simplest way to proceed is to note that the states derived from the anti-Pfaffian are just the particle-hole conjugates of those derived from the Pfaffian. It follows that these states-which we will call $\Psi_{\text {apf,hi }}^{m}$ and $\chi_{\text {apf,hi }}^{m}$-are necessarily Abelian and have filling fractions $(8 m+1) /(16 m$ $+1)$ and $(8 m-2) /(16 m-3)$, respectively. The simplest states (with $m=1$ ) occur at 9/17 and 6/13.

If one considers higher levels in the hierarchy as above, one can construct a state with any odd denominator filling 
fraction $\nu$ with $11 / 24<\nu<17 / 32$. As in the case of the Pfaffian, all of these states have the same quasiparticle charges and statistics as usual Abelian hierarchical states at the same filling fraction. However, they are distinct from the usual Abelian hierarchical states-as they have different thermal Hall conductance.

While these states are distinct from the usual Abelian hierarchical states, it is not clear that they are distinct from the hierarchical states derived from the Pfaffian state. For example, the $\nu=8 / 17$ state derived from the anti-Pfaffian $6 / 13$ state appears to have all the same properties as the Pfaffian derived $\nu=8 / 17$ state. Specifically, the two states have the same quasiparticle charges, statistics, and the same thermal Hall conductance.

\section{CANDIDATE STATES FOR A QUASIHOLE WIGNER CRYSTAL}

In this section we consider the problem of a quasihole Wigner crystal. We construct two candidate states for this system using an approach similar to the incompressible liquid case.

More specifically, the problem we wish to consider is the following. Take a FQH system whose ground state at half filling is the Pfaffian state and suppose that the filling is slightly less than $1 / 2$ so that there is a finite density of quasiholes. Suppose further that these quasiholes form a Wigner crystal (e.g., a triangular lattice). For conceptual simplicity, assume the quasiholes are pinned at the lattice sites so that there are no phonon modes at low energies. Because of the quasiholes' non-Abelian statistics, there will be $2^{N-1}$ nearly degenerate states for a lattice of $2 N$ quasiholes. While the splitting between these states vanishes in the limit of infinitely large lattice spacing, it will be nonzero for any finite sized lattice. Thus, a particular linear combination of the $2^{N-1}$ low-lying states will be selected as the ground state. We would like to understand what kind of ground states can occur in this system and what properties the associated FQH states have-e.g., quasiparticle statistics, thermal Hall conductance, etc.

As before, our approach will be to propose candidate ground states for this system and then to analyze their properties. Such ground states are specified by vectors with $2^{N-1}$ components. As before, the CFT ansatz suggests two natural vectors with $2^{N-1}$ components. The first is defined by

$$
\Psi_{\alpha}=\left\langle\bar{\sigma}\left(\bar{w}_{1}\right) \cdots \bar{\sigma}\left(\bar{w}_{2 N}\right)\right\rangle_{\alpha},
$$

where $w_{1}, \ldots, w_{2 N}$ are the positions of the pinning sites (which we have assumed form a triangular lattice). This state is closely related to the series of hierarchical states $\Psi^{m}$. Similarly, the second candidate state is closely related to the series of hierarchical states $\chi^{m}$. This state is given by

$$
\chi_{\alpha}=\left\langle\sigma\left(w_{1}\right) \cdots \sigma\left(w_{2 N}\right)\right\rangle_{\beta} R_{\beta \alpha} .
$$

Unlike the hierarchical states $\Psi^{m}$ and $\chi^{m}$ we do not have any arguments for why these states may be favored energetically. In fact we do not know any Hamiltonian for which they are the exact ground state. Nevertheless, we will assume that they are physical ground states and see what their properties are.

To derive these properties, we write out the wave functions for $\Psi, \chi$ in terms of the electron coordinates $\{z\}$. We have

$$
\begin{aligned}
\Psi(\{z\})= & \sum_{\alpha} \Psi_{\alpha} \cdot \Phi_{\alpha, \mathrm{qh}}(\{z\}) \\
= & \left\langle\sigma\left(w_{1}, \bar{w}_{1}\right) e^{i / \sqrt{8} \phi\left(w_{1}\right)} \cdot \psi\left(z_{1}\right) e^{\left.i \sqrt{2} \phi\left(z_{1}\right) \ldots\right\rangle}\right. \\
& \times \exp \left(-\frac{1}{4 l^{2}} \sum_{i}\left|z_{i}\right|^{2}\right),
\end{aligned}
$$

where the second equality follows from the same reasoning as Eq. (16). Similarly, one can show that $\chi(\{z\})$ is given by the correlator

$$
\begin{aligned}
\chi(\{z\})= & \left\langle e^{i / \sqrt{8} \phi^{\prime}\left(w_{1}\right)+i / 2 \phi^{\prime \prime}\left(w_{1}\right)} \cdots e^{i \sqrt{2} \phi^{\prime}\left(z_{1}\right)} \cos \left(\phi^{\prime \prime}\left(z_{1}\right)\right) \cdots\right\rangle \\
& \times \exp \left(-\frac{1}{4 l^{2}} \sum_{i}\left|z_{i}\right|^{2}\right) .
\end{aligned}
$$

To proceed further, we make another bold assumption: we assume that the CFT approach is still applicable even though the quasiholes are fixed in space and are not free to move as in a normal hierarchical state. That is, we assume that quasiparticle excitations can still be constructed by inserting operators $\mathcal{O}$ into the above correlators and that the statistics of these quasiparticles can still be computed from the monodromy of these correlators. We would like to emphasize that this is a conjecture-and is on even less firm ground than usual applications of the CFT approach. Indeed, the validity of the CFT approach in this context likely depends on the specific choice of lattice. For example, one can imagine that on some lattices (particularly those with an even number of quasiholes per unit cell) the wave functions $\Psi, \chi$ may describe highly dimerized states where quasiholes pair up with a fixed partner in either the 1 or $\psi$ fusion channel. In this case, the CFT approach could break down in the same way that it fails for the Wigner crystal states that occur at large $m$ in the usual Laughlin series. By using the CFT approach we are implicitly assuming that this kind of dimerization scenario does not occur - at least on the triangular lattice.

We begin with the state $\Psi$. The allowed operators $\mathcal{O}$ are those which are local with respect to the electron operator $\psi e^{i \sqrt{2} \phi}$. A complete list of such operators (or more accurately equivalence classes of such operators) is given by $\left\{v e^{i n \phi / \sqrt{2}}\right\}$, where $v=1, \sigma(z, \bar{z}), \mu(z, \bar{z}), \psi$, and $n$ is integer or half integer depending on whether $v=1, \psi$ or $v=\sigma(z, \bar{z}), \mu(z, \bar{z})$. One can check that all of these can be generated from the elementary operator $\mathcal{O}=\sigma(z, \bar{z}) e^{i \phi / 2 \sqrt{2}}$ (and the electron operator). Thus, all the quasiparticles are composites of an elementary quasiparticle corresponding to $\mathcal{O}$.

Using the usual arguments, it is easy to check that the elementary quasiparticle carries charge $\pm e / 4$, and statistical phase $e^{i \pi / 8}$. In particular, the state $\Psi$ is Abelian. One can go further and compute the thermal Hall conductance. Since the CFT has one forward propagating Majorana mode, one backward propagating Majorana mode and one forward propagating boson, the thermal Hall conductance is $1 / 2-1 / 2+1=1$ 
in appropriate units. On the other hand, the electric Hall conductance is $1 / 2$ (like the parent Pfaffian state) since the quasiholes are all localized.

We would like to mention that these properties agree exactly with the strong pairing $\nu=1 / 2$ state. ${ }^{11}$ Thus, it appears that $\Psi$ is in the same universality class as the strong pairing state.

Next, consider the state $\chi$. In this case, one finds that all allowed operators are composites of the elementary operator $\mathcal{O}=e^{i\left(\sqrt{1 / 8} \phi^{\prime}\left(w_{1}\right)+\phi^{\prime \prime}\left(w_{1}\right) / 2\right)}$. One finds that the corresponding elementary quasiparticle/quasihole carries charge $\pm e / 4$ and statistical phase $e^{3 i \pi / 8}$. The thermal Hall conductance is 1 $+1=2$ in appropriate units. Comparing with the $331 \nu=1 / 2$ state $^{11,12}$ we conclude that $\chi$ is in the same universality class as this state.

Putting this all together we conclude that a lattice of localized quasiholes can naturally give rise to Abelian states. We have found two such candidates, $\Psi$, and $\chi$-with different quasiparticle statistics and thermal Hall conductance.

As we mentioned earlier, these candidate states agree well with Kitaev's study of quasihole lattices ${ }^{13}$ (see also Ref. 14 and in the disordered case, Ref. 15). In that work, the author described an exact solution of a triangular lattice of quasiholes with a nearest-neighbor interaction that favored the one fusion channel. His conclusion was that the ground state was Abelian and in the strong pairing phase-the same universality class as $\Psi$. One can also consider the same model but with a nearest-neighbor interaction that favors the $\psi$ fusion channel. Using the same approach as in Ref. 13, one finds that the ground state is again Abelian. Moreover, one can show that it is in the 331 phase-the same universality class as $\chi$. This comparison suggests that our candidate statesand our construction in general-is at least somewhat natural.

\section{CONCLUSION}

In this paper, we have investigated collective states that can arise from a finite density of non-Abelian $e / 4$ quasiholes in a magnetic field. We have focused on two types of collective states: incompressible liquids and Wigner crystals. In the incompressible liquid case, we have proposed a natural series of incompressible states $\Psi^{m}$. These states are good candidate ground states for a model where the quasiholes have shortrange repulsive two-body interactions and are in their lowest effective Landau level. The $\Psi^{m}$ are associated with hierarchical FQH states $\Psi_{\text {hi }}^{m}$ derived from the Pfaffian state. Interestingly, these hierarchical states-which occur at filling fraction $\nu=8 m /(16 m+1)$ —are Abelian quantum Hall states. We have also investigated incompressible liquids of $e / 4$ quasiparticles. In that case, we have proposed another series of incompressible states $\chi^{m}$. The resulting hierarchical states $\chi_{\mathrm{hi}}^{m}$ are again Abelian and occur at filling fraction $\nu=(8 m$ $-1) /(16 m-3)$.

In the Wigner crystal case, we have proposed two candidate ground states $\Psi, \chi$, closely related to the incompressible liquids $\Psi^{m}$ and $\chi^{m}$. We have analyzed the properties of these states and we have shown that these states are also Abelian. Our results can be compared with those obtained from a microscopic model ${ }^{13}$ of a quasihole lattice. It appears that the microscopic analysis agrees with the results presented hereand suggests the same two Abelian phases.

While all of the states we have constructed are Abelian, we would like to reiterate that this is not the only possibility. A finite density of non-Abelian quasiparticles need not always destroy the non-Abelian statistics in the Pfaffian state. As we discussed earlier, one can imagine a scenario as in Ref. 7 where the quasiparticles pair and form tightly bound charge $e / 2$ Abelian anyon molecules which in turn form a Laughlin-type state. The result is a hierarchical state with the same non-Abelian statistics as the Pfaffian state. One can also consider a similar scenario in the Wigner crystal case. Depending on the interactions, the quasihole lattice may dimerize, with pairs of quasiholes favoring the 1 (or $\psi$ ) fusion channel. Again, the result is a non-Abelian state (in fact, in the same universality class as the original Pfaffian state). One can consider this to be a crude rule of thumb: hierarchical or the Wigner crystal states composed out of $e / 4$ quasiparticles are non-Abelian if the quasiparticles pair, and Abelian otherwise.

A natural direction for future research would be to extend our analysis to general Read-Rezayi states. It would be particularly interesting to apply these methods to the problem of a Wigner crystal of Read-Rezayi quasiholes. Indeed, unlike the Pfaffian case, microscopic models of this system have not been solved exactly (except in the case of a one dimensional chain of quasiholes $\left.{ }^{23}\right)$. The approach outlined in this paper could suggest potential phases of this poorly understood system.

\section{ACKNOWLEDGMENTS}

We would like to thank Ady Stern and Chetan Nayak for useful discussions. This work was supported by the Microsoft Corporation, by NSF under Grants No. DMR-0541988 and No. DMR-05-29399, and by the Harvard Society of Fellows.
${ }^{1}$ G. Moore and N. Read, Nucl. Phys. B 360, 362 (1991).

${ }^{2}$ S. S. Lee, S. Ryu, C. Nayak, and M. P. A. Fisher, Phys. Rev. Lett. 99, 236807 (2007).

${ }^{3}$ M. Levin, B. I. Halperin, and B. Rosenow, Phys. Rev. Lett. 99, 236806 (2007).

${ }^{4}$ J. S. Xia, W. Pan, C. L. Vincente, E. D. Adams, N. S. Sullivan,
H. L. Stormer, D. C. Tsui, L. N. Pfeiffer, K. W. Baldwin, and K. W. West, Phys. Rev. Lett. 93, 176809 (2004).

${ }^{5}$ F. D. M. Haldane, Phys. Rev. Lett. 51, 605 (1983).

${ }^{6}$ B. I. Halperin, Phys. Rev. Lett. 52, 1583 (1984).

${ }^{7}$ P. Bonderson and J. K. Slingerland, Phys. Rev. B 78, 125323 (2008). 
${ }^{8}$ R. B. Laughlin, Phys. Rev. Lett. 50, 1395 (1983).

${ }^{9}$ J. K. Jain, Phys. Rev. Lett. 63, 199 (1989).

${ }^{10}$ B. Blok and X.-G. Wen, Phys. Rev. B 42, 8145 (1990).

${ }^{11}$ B. I. Halperin, Helv. Phys. Acta 56, 75 (1983).

${ }^{12}$ F. Haldane and E. Rezayi, Phys. Rev. Lett. 60, 1886 (1988).

${ }^{13}$ A. Kitaev (unpublished).

${ }^{14}$ E. Grosfeld and A. Stern, Phys. Rev. B 73, 201303(R) (2006).

${ }^{15}$ N. Read and A. W. W. Ludwig, Phys. Rev. B 63, 024404 (2000).

${ }^{16}$ N. Read and E. Rezayi, Phys. Rev. B 59, 8084 (1999).

${ }^{17}$ P. D. Francesco, P. Mathieu, and D. Senechal, Conformal Field Theory (Springer-Verlag, New York, 1997).
${ }^{18}$ N. Read, Phys. Rev. B 79, 045308 (2009).

${ }^{19}$ X.-G. Wen, Adv. Phys. 44, 405 (1995).

${ }^{20}$ C. Nayak and F. Wilczek, Nucl. Phys. B 479, 529 (1996).

${ }^{21}$ H. Georgi, Lie Algebras in Particle Physics (Perseus Books, Reading, MA, 1999).

${ }^{22}$ R. Dijkgraaf, C. Vafa, E. Verlinde, and H. Verlinde, Commun. Math. Phys. 123, 485 (1989).

${ }^{23}$ A. Feiguin, S. Trebst, A. W. W. Ludwig, M. Troyer, A. Kitaev, Z. Wang, and M. H. Freedman, Phys. Rev. Lett. 98, 160409 (2007). 